\title{
La investigación de la gobernanza en México y su aplicabilidad ambiental
}

\section{The research on governance in Mexico and its environmental applicability}

\author{
Nain Martínez \\ ILEANA ESPEJEL*
}

\begin{abstract}
Discussion over governance is increasing in environmental sciences because of a greater participation of stakeholders in environmental management. Nevertheless, the concepts surrounding the debate are often overlapping or unclear. The present study aims to add cohesion to the debate by contextualizing and clarifying relevant terms and definitions. For this end, we present a literature review overview of the evolution of the research on governance in Mexico (1995-2012). By doing so, we propose a new working definition of environmental governance, distinguishing this concept from governability and government.
\end{abstract}

Keywords: environmental governance, citizen participation, environmental issues, Mexico.

\section{Resumen}

La discusión sobre la gobernanza es cada vez más frecuente dentro de las ciencias ambientales debido a una mayor participación de los actores sociales en la gestión ambiental. No obstante, las definiciones que rodean el debate a menudo son poco claras. El presente estudio pretende contribuir a la cohesión del debate al contextualizar y clarificar los términos y definiciones pertinentes. Se presenta una revisión de la investigación sobre la gobernanza en publicaciones mexicanas (1995-2012), de este modo, se propone una nueva definición de trabajo de gobernanza ambiental, al distinguir este concepto del de gobernabilidad y gobierno.

Palabras clave: gobernanza ambiental, participación ciudadana, temas ambientales, México.

*Universidad Autónoma de California. Correo-e: nain.martinez@uabc.edu.mx; martinez.nain@ gmail.com, ileana.espejel@uabc.edu.mx; iespejel@gmail.com 


\section{Introducción}

El objetivo de esta investigación es hacer una revisión bibliográfica sobre los trabajos de la gobernanza en México para exponer su panorama general y, a partir de estos elementos, profundizar en el uso del concepto de la gobernanza en el sector ambiental. La finalidad es proponer una noción integradora de las diferentes perspectivas de investigación y explorar su alcance en la investigación ambiental en el contexto mexicano. Con esto, pretendemos realizar una aportación para clarificar el debate sobre el uso de la teoría de la gobernanza en lo ambiental y beneficiar a las futuras investigaciones en la materia.

En la década de los ochenta, el concepto de gobernanza (governance) era utilizado en estudios del sector privado en relación con una mayor interdependencia de la gestión corporativa de sus proveedores y clientes. Hasta 1989, el Banco Mundial adoptó el concepto para el ámbito gubernamental en El África subsahariana: de la crisis al desarrollo sustentable. Una perspectiva de largo plazo, donde se señalaba que los problemas sociopolíticos de las naciones africanas se debían a la incapacidad de sus gobiernos para dirigir las políticas públicas (Rodríguez-González, 2009).

Posteriormente, el concepto fue empleado por primera vez en el medio académico por Kooiman (1993) para describir cambios en el modelo tradicional de gobernar, que en su opinión transitaba de un proceso unidireccional (de los gobernantes a los gobernados) a otro multi-direccional donde los actores sociales influían en las decisiones del gobierno a través de una red de interacciones, lo cual generaba una mayor interdependencia entre las esferas pública y privada.

La gobernanza es definida como el proceso de dirección sociopolítica que incrementa las interacciones de los actores sociales y gubernamentales (Kooiman, 2003) debido a la adaptación del gobierno a condiciones de mayor complejidad social, económica y política (Peters y Pierre, 2005), lo cual deriva en redes interdependientes de actores con mayor grado de autonomía e influencia en el ciclo de las políticas públicas (Rhodes, 1996).

En la literatura existen diferencias en la interpretación de la gobernanza, sin embargo, las diferentes perspectivas teóricas comparten elementos como a) la poca capacidad del gobierno para imponer una agenda en las sociedades contemporáneas, b) el incremento de recursos por parte de actores sociales para actuar en la esfera pública (legitimidad, financiamiento, información, tecnología, capital humano, entre otros), c) los procesos de colaboración, vinculación, codirección, coproducción, regulación e implementación compartida entre los actores sociales y el gobierno y, d) la dilución entre los límites de la esfera pública y privada (Aguilar-Villanueva, 2010; Kooiman, 1993; Prats, 2005; Peters y Pierre, 2005). 
La discusión que observamos en las lecturas sobre la gobernanza se centra en sus límites conceptuales; por un lado, desde una de las posiciones teóricas, la gobernanza explica la transición de un gobierno tradicional (jerárquico y centralizado) a un gobierno más abierto que interactúa con los actores sociales. En consecuencia, la gobernanza aglutina sus diferentes estadios (desde el gobierno tradicional hasta el relacional) (Kooiman, 2003).

En contraparte, otros autores argumentan que la gobernanza cobra sentido en las circunstancias contemporáneas, en las que se presenta una mayor participación de los actores sociales en los asuntos públicos; por lo tanto, distinguen entre una vieja gobernanza (old governance) y una nueva (new governance) (Rhodes, 1996). Estas posiciones se diferencian en que para algunos autores la gobernanza es un proceso gradual, mientras que para otros representa una ruptura con el gobierno tradicional. Además, en la teoría de la gobernanza, el papel del Estado es otro punto de discusión. Por ejemplo, Prats (2005) dice que el Estado conserva una posición central como el único actor con capacidad para validar los acuerdos que se generan entre los actores. En contraste, Filibi-López (2010) señala que hay una redefinición de los límites de la esfera política con el debilitamiento del Estado y el fortalecimiento de entidades sub y supranacionales.

En este sentido, Aguilar-Villanueva (2010) señala que la gobernanza es una respuesta a las nuevas tendencias en las políticas y administración públicas que buscan una relación más equilibrada entre el conocimiento académico y el político, una mayor participación de actores sociales y una revaloración de las capacidades gubernamentales y sociales; también responde a los cambios sociales, por ejemplo, Peters y Pierre (2005) explican que el interés en la gobernanza se debe a causas como la crisis financiera de la década de los ochenta, la cual generó la percepción de fracaso de los gobiernos, la liberalización de los mercados, la globalización, una mayor complejidad de las sociedades y nuevas tendencias en la gestión pública.

Por otro lado, pensamos que la problemática ambiental también fue un disparador de la necesidad de una mayor participación social en el proceso de toma de decisiones. En la Conferencia de Estocolmo de 1972, la comunidad internacional reconoció la problemática ambiental sentando las bases para su gestión, sin embargo se consideró que podría ser resuelta desde la aplicación de medidas técnicas. Fue a partir del Informe Brundtland de 1987 que se introdujo el concepto de sustentabilidad para integrar los componentes sociales y económicos en la gestión ambiental (Lezama, 2010). En él, se proponía que los problemas ambientales no sólo eran de índole ecológica, sino el producto de la interacción de las sociedades y sus procesos económicos con los ecosistemas (Foladori, 2002). 
En la actualidad, la problemática ambiental se ha convertido en una de las principales preocupaciones de la sociedad. En consecuencia, en la comunidad internacional existe un creciente interés en mejorar la política en este sector y sus instrumentos de gestión (Lezama, 2010).

Sin embargo, la política ambiental presenta peculiaridades frente a otras arenas de política. Por ejemplo, los grandes temas ambientales como el cambio climático y la pérdida de biodiversidad muestran que el diseño de marcos normativos y la implementación de políticas deben realizarse con una amplia incertidumbre en la información y, en ocasiones, la implementación puede generar costos económicos o sociales y producir conflictos entre sectores (Ludwig et al., 2001).

En gran medida, la especificidad de la política ambiental ha sido explicada por la complejidad de los sistemas ambientales en los que se pretende influir, donde confluyen procesos ecológicos, necesidades sociales, instituciones y relaciones culturales (Leff, 2000; Ludwig et al., 2001; Reyes-Orta et al., 2013). En este sentido, Funtowicz y Ravetz (1993) desarrollaron el concepto de ciencia postnormal donde se plantea una metodología para las condiciones contemporáneas en la que los factores son inciertos, hay valores en disputa, las decisiones políticas pueden ser urgentes para la sociedad y los riesgos derivados de estas decisiones son altos. En estas circunstancias, se presenta una inversión de la distinción tradicional entre hechos científicos objetivos y los valores subjetivos. Así, en los problemas ambientales las decisiones políticas están conducidas por valores que son objetivos y para los cuales los aportes científicos parecieran subjetivos.

En este contexto, se ha propuesto el uso de los procesos participativos para incrementar el conocimiento sobre los sistemas ambientales y mejorar la calidad de las decisiones políticas. Por ejemplo, Funtowicz y Ravetz (1993) sugieren que las decisiones políticas en materia ambiental se deberían formular en una comunidad extendida de iguales, compuesta por todos los actores afectados o interesados.

Así, desde la diversidad de perspectivas e intereses legítimos de los actores se podría realizar un aporte de hechos extendido. Esto permitiría incluir el conocimiento tradicional, los intereses políticos y la información académica al proceso de toma de decisiones. En consecuencia, la participación puede aportar beneficios como aumentar la comunicación entre los actores, disminuir los conflictos, incrementar la legitimidad política y, en general, aumentar la calidad y durabilidad de las decisiones políticas (Funtowicz y Ravetz, 1993; Morse et al., 2007).

En la política ambiental mexicana la participación ciudadana se ha institucionalizado a través de diversos mecanismos y órganos de consulta debido a que la interacción entre los actores sociales y políticos tiene 
implicaciones en el manejo de los recursos naturales (Bobadilla et al., 2013; Martínez y Chávez-Ramírez, 2014). Por este motivo existe un creciente interés en utilizar la teoría de la gobernanza en la investigación ambiental, sin embargo no encontramos una revisión integrada de ambos acercamientos teóricos.

\section{Metodología}

Se realizó una revisión de las investigaciones publicadas que utilizan la teoría de la gobernanza en México, se emplearon como criterios de selección a) publicaciones de autores mexicanos y b) publicaciones de autores extranjeros sobre México, para lo cual se efectuó primero una búsqueda de artículos académicos completos entre el periodo de 1995-2012 disponibles en la base de datos Ebsco Host hasta febrero de 2012; se utilizaron como motor de búsqueda las palabras governance, gobernanza y México; fueron discriminados los textos que no hacían referencia directa a México o la teoría de la gobernanza; a continuación se desarrolló una búsqueda de publicaciones sobre este concepto en las principales bibliotecas, librerías y casas editoriales del país; posteriormente se realizó un análisis exploratorio a partir del cual se definieron las variables del estudio para hacer la revisión (cuadro 1). Para finalizar, se eligieron las publicaciones con el tema ambiental con el fin de hacer una revisión más profunda.

\section{Resultados}

La muestra de la revisión bibliográfica consistió en 75 datos que corresponden a 60 artículos de investigación y 15 textos provenientes de libros (libros y capítulos) (figura I). En los libros se encontraban publicadas principalmente las investigaciones teóricas y en los artículos académicos las investigaciones aplicadas y los estudios. Como es obvio, encontramos que $95 \%$ de los autores que realizan investigaciones en instituciones mexicanas y latinoamericanas publican en español y son quienes realizan la mayor parte de las aportaciones teóricas. Por otra parte, también por obviedad, los autores de instituciones estadounidenses y europeas publican en inglés pero sus aportaciones son principalmente estudios de caso.

De los estudios de caso, la temática ambiental es la de mayor amplitud (40\%) seguida de los estudios sobre aspectos económicos (16\%). Las investigaciones realizadas a escala supranacional $(24 \%)$ corresponden a la temática de relaciones internacionales. En la escala nacional la temática dominante versa sobre las instituciones y gestión pública (30\%), seguida de economía y ambiente (25\%). De los estudios realizados a escala subnacional, 59\% corresponde a investigaciones ambientales. 


\section{Cuadro 1 \\ Variables utilizadas en la revisión del concepto gobernanza en México}

\begin{tabular}{|c|c|c|}
\hline Variable & Criterio & Categoria \\
\hline Publicación & Tipo de publicación & $\begin{array}{l}\text { Artículos publicados en revistas académicas. } \\
\text { Libro o capítulo. }\end{array}$ \\
\hline Investigación & Tipo de investigación & $\begin{array}{l}\text { Investigaciones teóricas sobre la gobernanza. } \\
\text { Investigaciones prácticas que utilizan el } \\
\text { concepto de gobernanza. }\end{array}$ \\
\hline Idioma & Idioma de publicación & $\begin{array}{l}\text { Publicaciones escritas en inglés. } \\
\text { Publicaciones escritas en español. }\end{array}$ \\
\hline Autor & Nombre del autor & Nombre del primer autor. \\
\hline Año & $\begin{array}{l}\text { Año de la investiga- } \\
\text { ción }\end{array}$ & Año de publicación. \\
\hline Título & Título de investigación & Título de la publicación. \\
\hline Revista & Nombre de la revista & $\begin{array}{l}\text { En el caso de los artículos, revista en que fueron } \\
\text { publicados. }\end{array}$ \\
\hline Escala & $\begin{array}{l}\text { En los estudios de } \\
\text { caso, escala de la inves- } \\
\text { tigación }\end{array}$ & $\begin{array}{l}\text { Investigaciones a nivel país. } \\
\text { Investigaciones locales o regionales. } \\
\text { Investigaciones a nivel país en relación con una } \\
\text { o más naciones. }\end{array}$ \\
\hline \multirow[t]{8}{*}{ Temática } & $\begin{array}{l}\text { En los estudios de } \\
\text { caso, temática domi- } \\
\text { nante de las publica- } \\
\text { ción }\end{array}$ & $\begin{array}{l}\text { Investigaciones sobre empresas y relaciones } \\
\text { de negocios. } \\
\text { Investigaciones sobre la gestión de recursos } \\
\text { naturales. }\end{array}$ \\
\hline & & $\begin{array}{l}\text { Investigaciones sobre reformas y cambios en la } \\
\text { gestión pública. }\end{array}$ \\
\hline & & Investigaciones sobre el sector salud. \\
\hline & & Investigaciones sobre el sector educativo. \\
\hline & & Investigaciones sobre la gestión urbana. \\
\hline & & $\begin{array}{l}\text { Investigación sobre las relaciones de México } \\
\text { con uno o más países. }\end{array}$ \\
\hline & & $\begin{array}{l}\text { Investigaciones sobre los sectores cultural y } \\
\text { científico. }\end{array}$ \\
\hline & & $\begin{array}{l}\text { Investigaciones sobre organizaciones de la } \\
\text { Sociedad Civil. }\end{array}$ \\
\hline País & País de publicación & Nombre del país. \\
\hline Institución & $\begin{array}{l}\text { Nombre de institución } \\
\text { de investigadores }\end{array}$ & Nombre de la institución. \\
\hline
\end{tabular}

Fuente: elaboración propia. 


\section{Figura I}

\section{Proporción de tipos de publicaciones que tratan la teoría de la gobernanza en México}

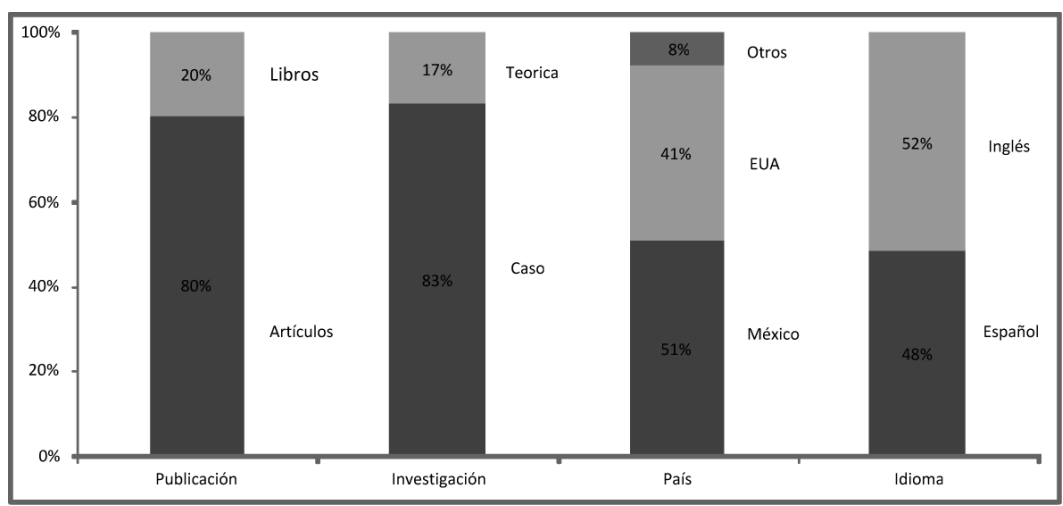

Fuente: elaboración propia.

Estos datos evidencian que la teoría de la gobernanza en México ha sido utilizada para estudiar procesos que van desde el ámbito internacional hasta las escalas regionales y locales. Por otra parte, si bien la teoría de la gobernanza es utilizada en una gran variedad de áreas de investigación como el análisis de instituciones públicas o las relaciones económicas, el sector ambiental presenta diversos procesos de interés para este cuerpo teórico que van desde la interrelación entre actores sociales y gubernamentales en el manejo de recursos naturales hasta los cambios que ha presentado la política ambiental en los últimos años.

Los autores más prolíficos son Aguilar-Villanueva (2006 y 2010), quien por extensión y profundidad ha realizado la mayor aportación sobre la discusión teórica de la gobernanza en México; Paz-Salinas (2007 y 2008) quien exploró las tensiones entre múltiples actores en el desarrollo de acuerdos públicos para el manejo de recursos naturales, y Basurto (2005 y 2008) quien investigó la auto-organización en el aprovechamiento de recursos pesqueros de la comunidad seri en el golfo de California. Además, Paré y Fuentes (2010) quienes indagaron, sobre la implementación de la Reserva de la Biosfera de los Tuxtlas, el papel de los diferentes actores, sus conflictos y relaciones. 


\subsection{Revisión de la teoría de la gobernanza en México}

\subsubsection{La crisis del Estado burocrático}

Después de la Segunda Guerra Mundial los Estados tuvieron un crecimiento sin precedentes en el tamaño de su burocracia y áreas de intervención de la vida social; para lograrlo requirieron un aumento constante en su poder y recursos provenientes de la sociedad a través de los impuestos. Los Estados burocráticos desarrollaron el corporativismo para manejar a los grupos de interés y se legitimaron con el incremento en el bienestar de la población (Filibi, 2010); pronto los gobiernos se vieron sin capacidad de respuesta a las crecientes demandas sociales y, en la década de los setenta, entraron en crisis las sociedades industrializadas debido al desequilibrio hacendario, generando retroceso o estancamiento económico por el incremento de la inflación (Aguilar-Villanueva, 2006).

En latinoamérica, en la década de los ochenta, el modelo económico de sustitución de importaciones, del que México era su principal exponente, se encontraba en crisis porque disminuía el ritmo de crecimiento económico y, para dar respuesta a las demandas sociales y preservar la estabilidad de los regímenes autoritarios, los gobiernos solicitaban préstamos internacionales que se convirtieron en deudas impagables (Bassols, 2011).

El gobierno había sido concebido como el único actor organizado y con recursos suficientes para guiar a una sociedad que se percibía caótica y sin capacidad de organización. Sin embargo, las constantes crisis económicas y la incapacidad de generar respuestas satisfactorias pusieron de manifiesto que el gobierno también podía ser un factor de desorganización y que no todas sus políticas eran eficaces (Rojo-Salgado, 2010).

Aguilar-Villanueva (2010) señala que para solventar la crisis del Estado se produjeron dos tipos de respuestas. Por un lado se ahondó en cómo abastecer al gobierno de los instrumentos directivos para cumplir sus funciones a través del estudio de la gobernabilidad y, por otro lado, se analizaron las limitaciones del actuar del gobierno enfocando la atención en el proceso de gobernación más que en el gobierno como sujeto. Se hicieron distinciones entre las funciones de gobierno y el actuar del gobierno, reconociendo que no siempre ni en todas las esferas, el Estado poseía todos los recursos para dirigir con eficiencia. Asimismo, se reconoció que en la sociedad existían actores con recursos económicos, tecnológicos, informativos y de legitimidad que podían ser empleados para generar mejores resultados.

El proceso de gobernación se encontraba homologado al gobierno y diferenciar entre ambos generó un profundo cambio conceptual, princi- 
palmente porque los antiguos instrumentos de gobierno implementados de manera directa por sus burocracias ya no eran suficientes para aprovechar las oportunidades y solventar los problemas que requerían la cooperación entre gobiernos y la concertación con actores subnacionales y transnacionales.

En este proceso de diferenciación se reconocía que había condiciones contemporáneas particulares, como la globalización económica, la deslocalización de empresas y capitales, la mayor permeabilidad de las fronteras nacionales por flujos migratorios, la articulación de redes de organizaciones sociales y empresariales de carácter transnacional y sociedades más complejas, diversas y diferenciadas. En tales circunstancias, el gobierno debía replantearse su papel en la sociedad y transformarse de quien implementa la política a una entidad de dirección, vinculación y articulación de los esfuerzos sociales (Aguilar-Villanueva, 2006; Porras, 2011).

\subsubsection{La gobernanza, ¿distinta a la gobernabilidad y el gobierno?}

El concepto en inglés governance ha tenido problemas para su traducción al idioma espańol, por un lado se han empleado diferentes vocablos como gobernancia, gobernación y gobernanza y, por el otro lado, algunos autores lo han entendido como sinónimo de gobierno y gobernabilidad. Sin embargo, en 2002 un grupo de académicos reunidos en Salamanca determinó que gobernanza era la traducción adecuada al español y diferentes teóricos de habla hispana se han dado a la tarea de delimitarlo (Concepción-Montiel, 2010; Aguilar-Villanueva, 2010; Porras, 2011).

El concepto de gobierno (government) hace referencia a la organización que representa al Estado, estructurada con base en poderes generales y leyes para ordenar y dirigir a la sociedad. La gobernabilidad (governability) trata de las capacidades del gobierno para cumplir sus funciones (figura II), por lo que centra su interés en cómo dotar al gobierno de las capacidades institucionales, económicas y de legitimidad para guiar a la sociedad. Los estudios de gobernabilidad se centran en al ámbito gubernamental (Aguilar-Villanueva, 2010).

El concepto de gobernanza se enfoca al proceso de gobernación e indaga en el gobierno como actor, pero va más allá e incluye a los sectores sociales. La gobernanza abarca tanto al gobierno como a la gobernabilidad, ya que se requiere de un gobierno eficiente pero reconoce que, aún en la situación de un gobierno con recursos suficientes, en la realidad contemporánea de mayor complejidad sociopolítica se requiere de la cooperación de los actores sociales (Aguilar-Villanueva, 2006). 


\section{Figura II \\ Elementos para diferenciar los conceptos de gobierno, gobernabilidad y gobernanza}

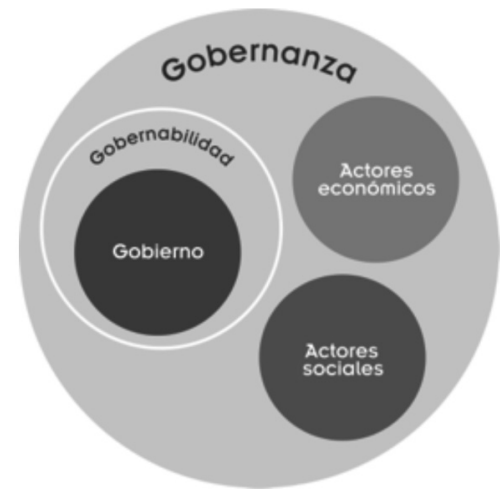

Fuente: elaboración propia.

\subsubsection{El concepto de gobernanza}

El concepto de gobernanza explica que cuando los gobiernos presentan problemas económicos o de legitimidad para cumplir con sus funciones, éstos se fragmentan y producen políticas públicas donde el sector privado, las organizaciones sociales y la sociedad en general cobran un papel relevante, convirtiéndose en objetos y sujetos de la política (Porras, 2011).

Aguilar-Villanueva (2006) señala que la crisis del Estado burocrático permitió diferenciar entre la acción del gobierno y la gobernación como proceso de conducción social, lo cual era impensable décadas atrás en el apogeo del Estado. De esta manera, la gobernanza es un concepto de postcrisis que se nutre de dos raíces, por un lado retoma la experiencia de la crisis del gobierno y por el otro lado perfila, a partir de la realidad contemporánea, un futuro que integra actores sociales con mejor capacidad organizativa, mayores recursos a su disposición y mayor interdependencia entre ellos.

La gobernanza es definida como un nuevo estilo (o proceso) de gobernación diferente al esquema jerárquico y centralizado del gobierno tradicional en el que la burocracia y actores sociales interactúan en redes decisionales caracterizadas por procesos de negociación, cooperación y coproducción en la definición de las políticas públicas (Bassols, 2011).

Aguilar-Villanueva (2010) señala que, en un primer momento, la gobernanza fue un concepto descriptivo que permitió a los académicos analizar nuevos procesos de interacción entre gobierno y actores sociales. 
Después, el concepto cobró un sentido normativo al convertirse, para algunas organizaciones internacionales, en representante de las mejores prácticas de gobierno; por lo que se recomendaba a los gobiernos implementar mecanismos cooperativos con sus sociedades. Fue hasta que se delimitaron las causas que condujeron al nuevo proceso de gobernación y se explicaron los efectos que producía en la sociedad, que la gobernanza cristalizó en un concepto teórico.

Peters (2004) indica que teóricamente se plantea un escenario de gobernanza sin gobierno, pero en la implementación el gobierno sigue ocupando un papel relevante. Sin embargo, el papel de la burocracia se modifica en relación con la administración pública tradicional y reconoce los siguientes elementos de cambio:

- La burocracia pierde en gran medida su autosuficiencia debido a que las decisiones deben ser discutidas y negociadas con los actores sociales para conseguir su cooperación.

- Los esquemas de gobernanza facultan a la sociedad y a los niveles inferiores de la burocracia para tomar decisiones, debilitando la frontera entre ambas esferas.

- Debido a que la administración de servicios públicos atañe al gobierno, pero también al sector social, la rendición de cuentas se transforma en un proceso colaborativo.

- Aunque existen marcos legales para la implementación de políticas públicas, en la gobernanza se aplican diferentes versiones de éstas, dependiendo del contexto y los procesos de negociación.

- La función pública disminuye su preeminencia en la provisión de productos de políticas debido a que actores no gubernamentales también pueden proporcionarlos. Con esto se abre paso a una extensa variedad de esquemas de colaboración entre el gobierno y personas provenientes de organizaciones sociales, la iniciativa privada y los usuarios.

- La gobernanza plantea esquemas más amplios para el desarrollo de la democracia en la administración pública debido a que involucra a los actores no-gubernamentales en la toma de decisiones y reconoce otros espacios de expresión del deseo social, por ejemplo, foros y consultas.

- Disminuye la participación de la administración pública en la implementación directa de la política. En este sentido, la burocracia vuelca su atención al manejo de relaciones con los sectores no-gubernamentales con el fin de gestar los procesos de participación, supervisar la implementación de tareas desarrolladas por los actores sociales, generar asociaciones y hacer subcontrataciones. 
En resumen, la gobernanza plantea trasformar la gestión pública de una burocracia centralizada y jerárquica, que actúa de manera uniforme, a una administración pública más descentralizada, abierta y flexible, y con mayor participación de los funcionarios públicos de menor nivel en interacción con la sociedad (Pardo, 2004).

\subsection{Estado de las investigaciones aplicadas y estudios de caso}

Las áreas de estudio abordadas desde diversas temáticas por el concepto de gobernanza tienen como factor común su origen en la integración de México al proceso de globalización, lo cual ha generado cambios reconocibles a diferentes escalas territoriales (cuadro 2). En el ámbito global se observa el impacto que tienen a nivel nacional las decisiones tomadas en otros países, la interdependencia entre naciones en la gestión de problemas en común, la construcción de mecanismos de cooperación transnacional, un papel más relevante de los actores económicos y sociales en la política global que incluso llegan a generar sus propias regulaciones y estándares de certificación (Coleman, 2007), y las nuevas e intrincadas relaciones entre gobiernos, el sector privado y las organizaciones sociales. Por ejemplo, una certificación internacional promueve mejoras laborales o el uso de nuevas tecnologías (Foladori, 2009; Rodríguez-Garavito, 2008).

En el ámbito nacional se indaga en las reformas emprendidas en diferentes sectores como el eléctrico, la política rural y la política electoral para otorgar una mayor participación al sector económico y social en áreas históricamente dominadas por el control gubernamental. Las reformas propiciaron la reconfiguración de poder entre actores y su adaptación a las nuevas circunstancias (Fox, 1995; Monsiváis, 2006).

En el ámbito local se explora la adaptación del municipio, las ciudades y las comunidades a los cambios respaldados a nivel nacional y global, a través de los mecanismos de participación y colaboración con el sector económico y la sociedad organizada, así como las nuevas funciones que cobran los actores sociales en la definición de políticas y la provisión de bienes y servicios públicos (Guarneros-Meza, 2011; Moreno-Mena, 2010).

\subsection{Revisión de los estudios ambientales que utilizan el concepto de gobernanza}

El grupo de textos que utilizan el concepto de gobernanza en la investigación ambiental esboza procesos como la autorganización comunitaria para el aprovechamiento de recursos naturales, la transformación de las instituciones y la política ambiental, la influencia de la globalización a través de nuevos mecanismos de regulación no gubernamentales y la ar- 


\section{Cuadro 2}

\section{Fenómenos analizados en México desde la teoría de la gobernanza}

\begin{tabular}{|c|c|}
\hline Temática & Ámbitos de estudio \\
\hline \multirow[t]{2}{*}{$\begin{array}{l}\text { Instituciones } \\
\text { y gestión pública }\end{array}$} & $\begin{array}{l}\text { Las reformas emprendidas a raíz de la integración de México al } \\
\text { proceso de globalización para otorgar una mayor participación } \\
\text { al sector económico y social. }\end{array}$ \\
\hline & $\begin{array}{l}\text { La construcción de mecanismos de cooperación regionales deri- } \\
\text { vados del Tratado de Libre Comercio de América del Norte. }\end{array}$ \\
\hline \multirow[t]{3}{*}{ Economía } & $\begin{array}{l}\text { La evolución de los actores económicos a las nuevas circunstancias } \\
\text { globales. }\end{array}$ \\
\hline & $\begin{array}{l}\text { La interacción entre el sector privado con nuevos esquemas de } \\
\text { regulación de origen no-gubernamental. }\end{array}$ \\
\hline & El papel de las ciudades en las redes económicas transnacionales. \\
\hline Gestión urbana & $\begin{array}{l}\text { Los nuevos procesos de toma de decisiones a nivel urbano para } \\
\text { impulsar proyectos de infraestructura o elaborar políticas públi- } \\
\text { cas en colaboración con actores sociales. }\end{array}$ \\
\hline \multirow[t]{3}{*}{ Educación } & Cambios en las relaciones entre los diferentes niveles de gobierno. \\
\hline & $\begin{array}{l}\text { Relaciones de interdependencia entre el gobierno y las institu- } \\
\text { ciones educativas. }\end{array}$ \\
\hline & $\begin{array}{l}\text { Mayor participación de las comunidades en las escuelas y la } \\
\text { política educativa. }\end{array}$ \\
\hline \multirow[t]{2}{*}{$\begin{array}{l}\text { Relaciones } \\
\text { internacionales }\end{array}$} & $\begin{array}{l}\text { Interdependencia de los gobiernos en la elaboración de políticas, } \\
\text { debido la aparición de redes de políticas públicas transnacionales. }\end{array}$ \\
\hline & $\begin{array}{l}\text { Insuficiencia de las políticas unilaterales en temas complejos como } \\
\text { la migración. }\end{array}$ \\
\hline \multirow[t]{2}{*}{$\begin{array}{l}\text { Organizaciones } \\
\text { sociales }\end{array}$} & $\begin{array}{l}\text { El papel de las Organizaciones de la Sociedad Civil (osc) en la } \\
\text { gestión de políticas públicas. }\end{array}$ \\
\hline & $\begin{array}{l}\text { Las estrategias de colaboración entre gobierno y sociedad civil en } \\
\text { la provisión de servicios. }\end{array}$ \\
\hline \multirow[t]{2}{*}{ Salud } & $\begin{array}{l}\text { El proceso de descentralización de la política de salud del gobier- } \\
\text { no federal hacia las entidades federativas. }\end{array}$ \\
\hline & $\begin{array}{l}\text { La coordinación entre México, Estados Unidos y Canadá para } \\
\text { enfrentar problemas de salud regionales. }\end{array}$ \\
\hline \multirow[t]{2}{*}{ Ciencia y cultura } & $\begin{array}{l}\text { Las tensiones entre diferentes actores a nivel global en la discusión } \\
\text { sobre la reglamentación de nuevas tecnologías. }\end{array}$ \\
\hline & $\begin{array}{l}\text { El escaso margen de dirección de la política cultural guberna- } \\
\text { mental y la necesidad de generar sinergias con actores sociales. }\end{array}$ \\
\hline
\end{tabular}

Fuente: elaboración propia. 
ticulación de redes transnacionales y, en general, el impacto de la política ambiental en la sociedad.

Se enfocan en temáticas como el manejo forestal (según nuestra contabilización corresponde a 24\%), costero (16\%), la gestión del agua (24\%), las Áreas Naturales Protegidas (ANP) (28\%) y aspectos relacionados con el aprovechamiento de recursos naturales en el medio rural (8 por ciento).

Sobre el manejo forestal, Klooster (2006) indica que la red de productores de madera compuesta por pobladores, gestores, agencias gubernamentales y organizaciones de la sociedad civil han incorporado mejores prácticas de gestión del recurso debido al creciente interés, a nivel mundial, de la certificación de la producción de madera a través de las osc. Sin embargo, cuestiona los beneficios reales que genera a los campesinos.

Wilshusen (2010) agrega que en México los productores rurales se han adaptado a la privatización de los recursos naturales y sostiene que dicha capacidad de resistencia constituye un elemento cultural previo a las reformas neoliberales. Asimismo, Racelis y Barsimantov (2008) analizan la estrategia de gestión de palizada ${ }^{1}$ como un recurso alternativo a maderas de mayor valor comercial y encuentran que la estrategia de gestión es incorrecta al no incorporar la información local sobre el recurso que podría servir para su manejo sustentable.

Barsimantov (2009) visualiza el papel más activo de actores no-gubernamentales en la asistencia técnica y financiamiento de la silvicultura. Finalmente, Antinori y Rausser (2007) estudian la capacidad de las comunidades en la toma de decisiones sobre sus recursos y encuentran que el uso de foros y espacios deliberativos socializa la política y fomenta las capacidades sociales para la participación, lo que resulta en un mayor grado de conservación de los bosques.

En los estudios del manejo costero se evalúan los factores de degradación del golfo de California, se encuentran como focos de atención de la política ambiental diversos temas de índole nacional como la contaminación, la creación de ANP marinas y una regulación más estricta de la pesca. Sin embargo, los esfuerzos de recuperación del ecosistema requieren de la colaboración internacional con Estados Unidos para incrementar el aporte de agua del río Colorado (Hyun, 2005). Por casos como este Rivera-Arriaga y Azuz-Adeath (2010) señalan la importancia de construir mecanismos de gobernanza internacional en las zonas costeras.

Por otro lado, Basurto (2005) estudia la organización de la comunidad seri en el golfo de California para aprovechar los recursos pesqueros de manera sustentable al margen de las instituciones gubernamentales. En

${ }^{1}$ Palizada: diversas especies de árboles pequeńos aprovechadas por su madera dura en el estado de Quintana Roo. 
una investigación posterior, Basurto (2008) describe los factores biológicos y ecológicos que influyen en la toma de decisiones de pescadores.

En los artículos que exploran la gestión del agua se estudian los cambios en la política de ésta en México como vía para la resolución de problemas en el manejo del recurso (Domínguez-Serrano, 2010; Tortajada, 2010). Según estos autores, dichas reformas han tendido a la coparticipación entre equipos técnicos, burocracia y los usuarios del recurso. Se publican estudios de caso sobre procesos participativos en la administración de distritos de riego y los consejos de cuencas (Palerm-Viqueira, 2009; Wilder, 2010), donde se propone que la gestión del agua bajo esquemas de gobernanza podría mejorar su administración (Salcido-Ruiz et al., 2010).

Dentro del grupo de investigaciones que tratan sobre ANP se exploran las tensiones entre la política de conservación y la población local. En este sentido, Paz-Salinas (2007), a partir de su investigación en el Corredor Biológico Chichinautzin, señala que no existen vías únicas para el establecimiento de una ANP debido a su diversidad social, cultural y política, por lo cual el reto para alcanzar los objetivos de conservación consiste en propiciar procesos de participación y construir las plataformas de negociación política y social para acceder a una sustentabilidad adaptativa.

Por otro lado, Guzmán-Chávez (2006) realiza un estudio comparativo entre la Reserva de la Biosfera Los Tuxtlas y la Reserva Extractivita Chico Mendes en Brasil y encuentra que los procesos históricos en ambos espacios determinan la política de conservación. Además, señala cómo factores externos de índole transnacional, cómo las fluctuaciones en el precio del café, pueden modificar la viabilidad de proyectos de desarrollo sustentable consensuados dentro de una reserva.

También, en la Reserva de la Biosfera Los Tuxtlas, Paré y Fuentes (2010) describen la complejidad del espacio debido a la diversidad de actores, tales como la burocracia de los tres niveles de gobierno, OsC extranjeras, centros de investigación, diferentes comunidades y sectores económicos. Señalan que los escasos resultados en la conservación de la naturaleza en áreas protegidas se deben a diversos factores como la falta de coordinación entre instituciones que operan en el área y las políticas que implementan, intereses políticos divergentes, conflictos entre actores y el fracaso de un proceso participativo. Este último, en el caso de Los Tuxtlas, no permitió reconocer nichos de oportunidad (por ejemplo las acciones de conservación voluntaria que realizaban algunas comunidades) para la gestión del área protegida.

De igual manera, en la Reserva de la Biosfera Sian Ka’an, Brenner (2010) realizó un amplio estudio sobre la gobernanza ambiental desde una óptica del poder de los actores y su posición sobre el manejo de los 
recursos; encontró asimetrías dentro de los actores locales, la inserción de actores externos como agencias gubernamentales y organizaciones no gubernamentales (ONG) internacionales, así como diferencias y contradicciones en los objetivos, estrategias y acciones de los actores.

Por esta razón señala que para el manejo adecuado del área se requieren mecanismos de mediación de interés en los que sea factible la conciliación y la coordinación. Este mismo autor, en un estudio posterior en la Reserva de la Biosfera Mariposa Monarca (Brenner, 2011), indaga sobre los órganos de participación y consulta establecidos para el manejo del área y encuentra que los resultados han sido deficientes debido a la complejidad política, la falta de legitimidad de los procesos participativos, la escasa participación de los actores sociales y los problemas de crimen organizado.

\section{Discusión}

\subsection{El estado del concepto de gobernanza en México}

Desde la década de los noventa se incorporó el concepto de la gobernanza en la investigación mexicana; en este periodo, la investigación sobre este tema ha sido fecunda en diversas disciplinas como las ciencias políticas, la administración pública, la sociología, la antropología, la economía y las ciencias ambientales. En la actualidad se cuenta con un corpus de conocimiento extenso y en crecimiento en el cual la gobernanza, más que objeto de estudio, se emplea como un concepto paraguas para describir e interpretar una extensa variedad de procesos sociopolíticos en diferentes escalas territoriales. Esta escalas pueden ser desde la organización de pequeños grupos en comunidades aisladas hasta la interdependencia de intrincadas redes políticas, sociales y comerciales en el ámbito transnacional.

La diversidad de las investigaciones expone la capacidad explicativa del concepto de una realidad contemporánea más compleja, convulsa e interdependiente. Todos los estudios de caso e investigaciones aplicadas tratan de instituciones, reglas, procesos, medios y mecanismos de gestión no detallados en los que los actores políticos, sociales y económicos experimentan nuevas formas de relación: el desmoronamiento de viejos nichos de poder, el desarrollo de nuevos espacios, procesos inacabados y, en general, una transición entre una situación estable a otra en construcción.

El desarrollo teórico retoma la discusión en el ámbito internacional sobre los límites del concepto. Es decir, la interpretación de que el proceso de gobernación es ejercido de manera centralizada y jerárquica por el Estado, denominado vieja gobernanza, y que la nueva gobernanza es la 
desarrollada por el gobierno en colaboración con actores sociales (AguilarVillanueva, 2006), bien, la posición que explica que el proceso de gobernación se encontraba homologado al gobierno y no es hasta que intervienen actores sociales que se distingue entre ambos y cobra sentido la gobernanza (Bassols, 2011).

A diferencia de la discusión internacional en la que se plantea una gobernación sin gobierno (Rhodes, 1996), en México, en general, se concuerda en que el gobierno conserva una posición como el único actor con capacidad para validar los acuerdos y procesos colaborativos entre los actores no-gubernamentales (Aguilar-Villanueva, 2010; Porras, 2011). Desde luego, existe una influencia del contexto histórico y social a partir del cual se realiza una aproximación teórica de la gobernanza. En México no se percibe factible o deseable una gobernación sin gobierno o un autogobierno. Sin embargo, en algunos estudios de caso se encuentran evidencias de auto-organización en comunidades tradicionales (Basurto, 2005 y 2008), lo cual contrasta con la posición teórica.

Las aportaciones teóricas en México son publicadas principalmente en libros y se realizan por autores mexicanos. En contraparte, las investigaciones aplicadas o estudios de caso se publican en revistas académicas en México y Estados Unidos, y en ellas participan autores de ambos países. Esta distinción es relevante debido a que el concepto de gobernanza (governance) tiene su origen en el idioma inglés y su inserción al español ha requerido no sólo de una traducción del vocablo sino de una traducción conceptual y su delimitación en relación a otros conceptos relacionados.

Este proceso de discusión ha quedado plasmado en los libros y no en los artículos académicos, lo cual, aunado a la discusión del concepto, ha generado que en diversas investigaciones aplicadas no se retomen las aportaciones teóricas endémicas y se utilice el concepto como sinónimo de gobierno o gobernabilidad. En este sentido, la gobernanza es una teoría del cambio que explica la transformación de nuestra la sociedad, el gobierno, sus instituciones y los roles ejercidos por nuevos actores. Por esta razón, al utilizar el concepto de gobernanza en una acepción tan amplia y no diferenciada del gobierno o de la gobernabilidad, se corre el riesgo de banalizar el concepto y perder su capacidad analítica de la fluida realidad contemporánea, que es en sí, su principal aportación.

\subsection{La inclusión del concepto de la gobernanza en la investigación ambiental}

El concepto de gobernanza en la investigación ambiental, en general, ha sido empleado para describir cuatro tipos de procesos: 1 ) de origen local, 
la auto-organización de comunidades tradicionales en el aprovechamiento de sus recursos naturales, lo cual es previo al proceso de globalización y reconfiguración del Estado a partir del cual emerge la teoría de la gobernanza. Sin embargo, el concepto resulta explicativo de las reglas establecidas por las comunidades en paralelo a la reglamentación gubernamental; 2) cambios a nivel nacional, reformas en la legislación de recursos naturales (agua, forestal, ejidal y protección ambiental) que implican, por un lado, la reconfiguración de actores en el ámbito local y nuevas dinámicas de interacción entre las burocracias, las comunidades y el sector privado y, por el otro, dan apertura a mecanismos de participación y consulta como medio para amortiguar la tensión social; 3) interdependencia global, los cambios en el aprovechamiento de los recursos naturales en comunidades locales debido a su adaptación a la globalización, apertura económica y la injerencia de actores transnacionales que dirigen la dinámica de las comunidades en paralelo al gobierno; 4) multinivel, se presenta por ejemplo en los estudios de caso sobre el manejo de ANP como un proceso hibrido que combina los tres anteriores, implica $a$ ) la inserción de agencias y organizaciones de la sociedad civil transnacionales al ámbito local con programas, políticas y recursos propios, $b$ ) el interés e injerencia de actores nacionales no-gubernamentales externos a las comunidades como osc, fundaciones e instituciones académicas, $c$ ) una complicada relación entre los tres niveles de gobierno y sus múltiples agencias, instituciones y políticas, d) la reconfiguración y cambios en el equilibrio de poder de actores sociales y comunitarios, y e) el surgimiento de órganos de participación y consulta como nuevos espacios con la potencialidad de articular acuerdos de manejo entre actores.

Consideramos que la apropiación del concepto de gobernanza en la investigación ambiental se debe en gran medida a que el medio ambiente, a diferencia de otras temáticas de investigación, es un espacio en el que los efectos de los procesos globales, nacionales y locales se asientan sobre el territorio y sus impactos repercuten en todos los actores regionales por lo que se convierten en causas de interés común. Los recursos naturales son un foco de tensión tangible donde es posible distinguir propietarios, usuarios, tasas de aprovechamiento y reglamentación formal e informal. Los actores instalados o no en el territorio y sus interacciones (colaboración, asociación, conflicto o neutralidad) son reconocibles debido a que su interés se encuentra sobre los recursos naturales.

En el caso de las ANP, éstas son áreas de estudio con actores multinivel y ejemplifican el alto grado de complejidad del manejo ambiental. Además, la legislación en la materia presenta una gama amplia de mecanismos de participación y consulta ciudadana. Por último, son espacios de diversidad cultural con marcadas asimetrías en el acceso a poder y recursos (tecno- 
lógicos, informativos, económicos y de legitimidad) entre actores. En resumen, la gobernanza como elemento clave de investigación desde la ciencia postnormal proporciona un marco teórico de referencia para explicar la creciente complejidad socioambiental; las transformaciones en el sector ambiental ejemplifican la fragmentación sociopolítica que se explica por la teoría de la gobernanza.

En los estudios de caso todos los autores coinciden en destacar la participación de actores no-gubernamentales en la definición de las políticas públicas, sin embargo, en la investigación ambiental se replica el traslape entre los conceptos de gobernanza, gobierno y gobernabilidad, que se traduce en tres cuestiones centrales: ¿Cuándo dejamos de hablar de la gobernanza y nos referimos al gobierno?, ¿Al estudiar el gobierno tradicional hablamos de otro tipo de gobernanza? y ¿en qué momento deja de ser un problema de gobernanza y tratamos un problema de gobernabilidad? A manera de ejemplificación, Brenner (2010), en su estudio sobre la gobernanza en ANP, utiliza por momentos el concepto para referirse a regímenes sobre los recursos naturales de prominencia estatal previos a esquemas de mayor apertura a la participación social y cuestiona si las plataformas multiactorales son esquemas eficaces de gobernanza cuando en ausencia de la participación social estaríamos haciendo referencia a un gobierno tradicional y no a un esquema de gobernanza. Rivera y Azuz-Adeath (2010) estudian la gobernanza del cambio climático, y si bien reconocen la importancia de la participación ciudadana, centran su interés en el sector gubernamental y retoman para su análisis, desde diversas teorías como el realismo y constructivismo, un enfoque teórico que podría corresponder a un estudio de gobernabilidad ambiental. En este contexto, nos pareció necesario insistir en la distinción, con mayor claridad, de los difusos límites conceptuales del concepto de la gobernanza en el medio ambiente.

\subsection{Una aproximación al concepto de gobernanza ambiental desde el contexto mexicano}

Con base en la diversidad de enfoques de investigaciones que utilizan el concepto de gobernanza en el sector ambiental, consideramos que éste es utilizado desde dos principales perspectivas: una normativa, centrada en incrementar la legitimidad de las decisiones gubernamentales a través de la participación ciudadana, y otra descriptiva de procesos de interacción entre actores sociales y gubernamentales.

En la perspectiva normativa, la teoría de la gobernanza es utilizada para proponer la implementación de un modelo de democracia participativa donde las decisiones gubernamentales adquirirían su legitimidad 
a través de la aceptación ciudadana (Domínguez-Serrano, 2010). De esta manera, la gobernanza es parte de una agenda política que pretende democratizar a la sociedad, transferir el poder de decisión a la ciudadanía e incrementar la eficiencia en el ejercicio gubernamental. Por lo tanto, las investigaciones que se realizan desde este enfoque centran su atención en las instituciones y las reformas que deben implementar para incorporar o mejorar los mecanismos de participación, con la finalidad de integrar los intereses de los actores sociales al proceso de toma de decisiones ( $\mathrm{Ri}$ vera-Arriga y Azuz-Adeath, 2010; Salcido-Ruiz et al., 2010).

Por otra parte, la posición descriptiva se aborda generalmente en los estudios de caso para estudiar los procesos sociopolíticos en el sector ambiental. De esta manera, la gobernanza es empleada para analizar procesos concretos de interacción entre actores sociales, económicos y gubernamentales en la gestión ambiental; estos procesos pueden desarrollarse a través de mecanismos informales, por ejemplo, con la elaboración de un reglamento informal por parte de actores comunitarios para el aprovechamiento de algún recurso natural (Basurto, 2005; Racelis y Barsimantov, 2008). También se presentan a través de mecanismos formales de participación incorporados recientemente en la legislación ambiental mexicana (Brenner, 2010; Paz-Salinas, 2007). Además, estos procesos de interacción pueden ubicarse desde el ámbito local hasta una escala trasnacional.

En este sentido, proponemos como concepto integrado de la gobernanza ambiental, al conjunto de instituciones, procesos y mecanismos, formales e informales, en los cuales interactúan actores interesados y afectados, tanto sociales, como económicos y gubernamentales en la gestión ambiental. Así, la gobernanza ambiental se presenta cuando en contextos de mayor complejidad ambiental, el gobierno carece de los recursos suficientes para dirigir la gestión ambiental y para incrementar su eficiencia, además requiere de la cooperación de actores sociales.

Por ejemplo, cuando el gobierno cuenta con información incompleta sobre las mejores posibilidades de aprovechamiento de recursos, su capacidad técnica es limitada; las instituciones requieren de la cooperación de la población local para su implementación; o bien, cuando los actores tienen objetivos y recursos propios (derechos sobre los recursos naturales, dinero, prestigio o legitimidad cultural e histórica) y éstos intervienen de manera paralela (cooperativa o complementaria al gobierno) en el manejo ambiental (Villada-Canela, 2013).

Finalmente, consideramos pertinente señalar que la investigación sobre la gobernanza ambiental se diferencia de otros enfoques analíticos, como la gobernabilidad o el gobierno, debido a que centra su atención en las interacciones entre los actores sociales y gubernamentales que in- 
fluyen en la gestión ambiental. En contraparte, la investigación sobre la política ambiental está más encaminada al funcionamiento de las leyes, políticas, instrumentos de políticas, programas y acciones que se desarrollan desde el ámbito gubernamental. En el caso de la gobernabilidad del sector ambiental, enfoca su atención en cómo fortalecer a las instituciones gubernamentales para el cumplimiento de sus objetivos (Aguilar-Villanueva, 2010).

\section{La aplicabilidad del concepto de la gobernanza ambiental}

El concepto propuesto de gobernanza ambiental es aplicable para el estudio de tres tipos de procesos que se pueden presentar en diferentes escalas. Primero, los procesos comunitarios de manejo de recursos, los cuales bajo la teoría de la gobernanza cobran un nuevo significado por la capacidad de los actores sociales para autorregularse; por ejemplo, una comunidad indígena que tiene reglas informales para el aprovechamiento de sus recursos naturales (Basurto, 2005). Segundo, procesos de organización y participación social que se realizan fuera de los mecanismos institucionales pero que influyen en el ámbito público: por ejemplo, una red de osc que desarrollan un proyecto de contraloría social, el cual logra influir en la toma de decisiones gubernamental. Tercero, la participación social que se realiza a través de mecanismos institucionales o previstos en la política ambiental; por ejemplo, una comunidad que colabora en la elaboración de un plan de manejo y su posterior implementación, los usuarios de un distrito de riego que se encargan de la administración del agua (Wilder, 2010) o diferentes actores sociales que participan en un consejo consultivo para la definición de la política en materia ambiental en su ciudad (figura III).

En el caso de los procesos de participación comunitaria en el manejo de recursos naturales, o de iniciativas de participación social autónoma, su existencia depende de las características históricas, culturales y políticas, por lo que no pueden ser generalizables o previsibles en el ámbito territorial (Brenner y De la Vega-Leinert, 2014).

Sin embargo, en el caso la participación que se realiza a través de mecanismos institucionales, es posible detectar una serie de mecanismos de participación en diferentes escalas, donde se presenta interacción de actores sociales y gubernamentales en la política ambiental (cuadro 3). Si bien estos mecanismos de participación no siempre funcionan, es posible esbozar una agenda de investigación donde precisamente se analice el funcionamiento de estos mecanismos; por ejemplo, la interacción entre los diferentes actores, los factores que influyen en la participación, la 


\section{Figura III}

Ejemplo de aplicación multiescalar del concepto de gobernanza ambiental en el manejo del agua

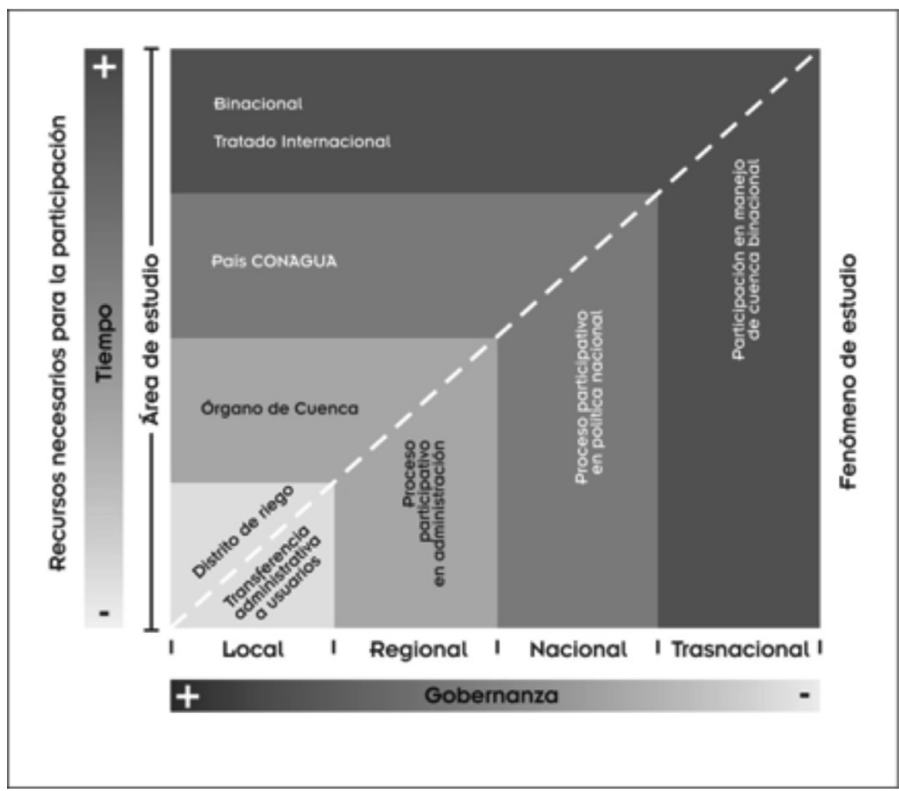

Fuente: elaboración propia.

capacidad que tienen los actores sociales para influir en la toma de decisiones y sus efectos en la gobernanza ambiental.

Por otra parte, en México la mayoría de las investigaciones de gobernanza en el medio ambiente se han realizado a nivel local y, en menor medida, regional y nacional. Además son escasos los estudios de los procesos participativos, lo que deja un amplio margen de investigación sobre el funcionamiento de los mecanismos de participación y consulta en diferentes escalas que utilicen la gobernanza ambiental como marco general de análisis.

\section{Conclusiones}

La inserción del concepto de gobernanza en México es reciente. Sin embargo, por su capacidad para explicar los cambios sociopolíticos de la realidad contemporánea, ha sido ampliamente utilizado en la investigación de diversas disciplinas como la administración pública, las ciencias políticas y las ciencias ambientales. En este sentido, la investigación sobre la gobernanza ha sido especialmente fructífera en la investigación ambiental. Esto se debe a que en las últimas décadas se han incorporado mecanismos 


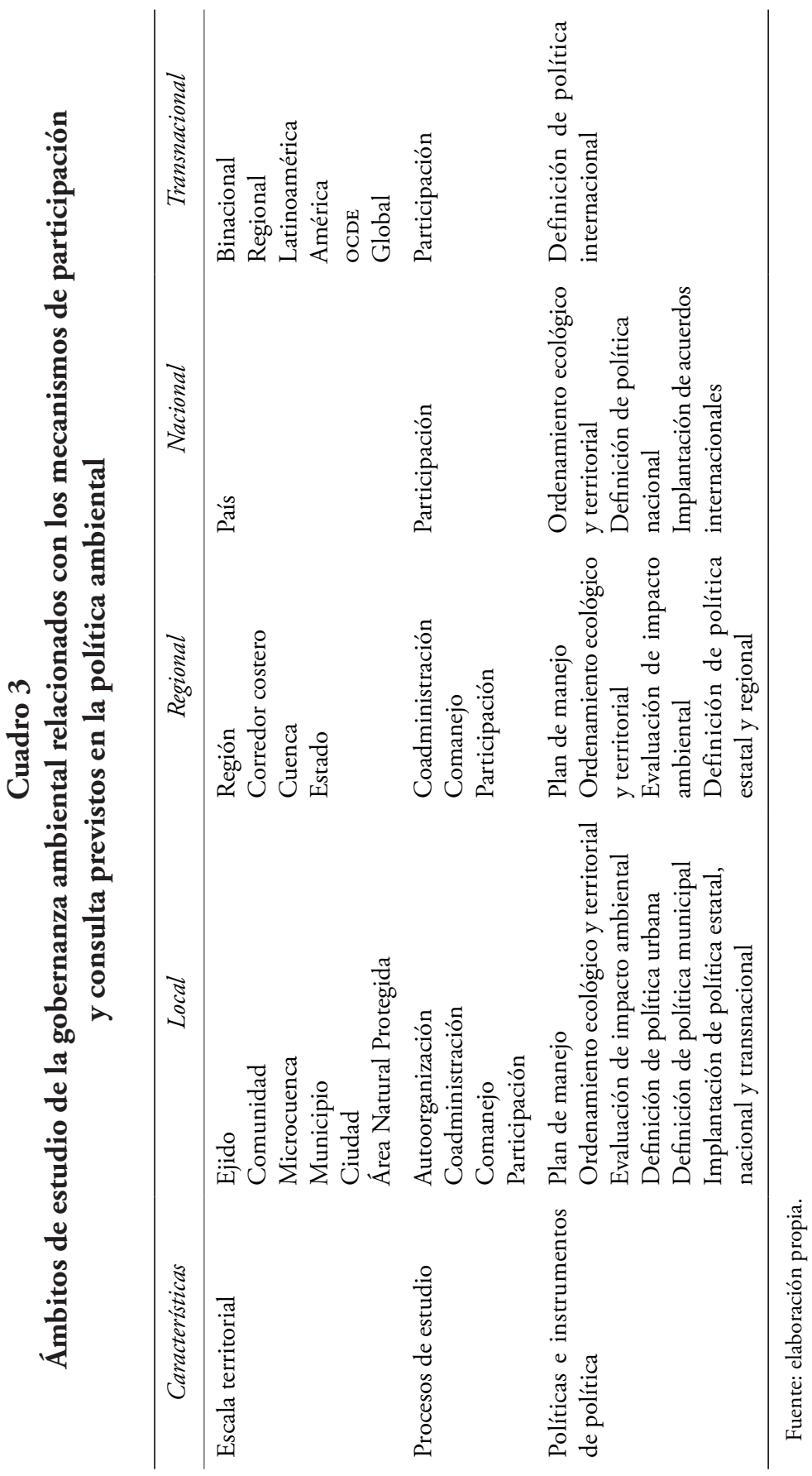


de participación ciudadana en este sector con la finalidad de integrar la complejidad ambiental al proceso de toma de decisiones, lo cual ha repercutido en una mayor interacción entre actores sociales y gubernamentales en la gestión ambiental.

La teoría de gobernanza aún se encuentra en discusión en sus límites. Por ejemplo, con relación al momento en que la gestión deja de ser un proceso basado en el gobierno tradicional para efectivamente incorporar a los actores sociales y convertirse en un proceso de gobernanza. No obstante, entre los teóricos existe un consenso sobre su originalidad en relación a otros acercamientos teóricos como la gobernabilidad y el gobierno. Así, esta revisión bibliográfica permitió exponer el panorama del desarrollo de la investigación de la gobernanza en México y con estos insumos construir un concepto de gobernanza ambiental y diferenciarlo de los enfoques analíticos.

El concepto propuesto de gobernanza ambiental aporta particularidad a la metodología de ciencia postnormal y a los estudios transdisciplinarios orientados a la investigación ambiental, y permite visualizar diversos ámbitos de estudio y procesos multiescalares para su implementación. Esto, relacionado a los mecanismos de participación y consulta previstos en la legislación ambiental mexicana, en áreas tan diversas y específicas como el manejo costero, la conservación de la biodiversidad, la gestión del agua y el uso de los recursos naturales.

Finalmente, es previsible que en México la investigación sobre la gobernanza ambiental continúe acrecentándose en la medida en que se analice la influencia de los procesos de interacción entre los actores sociales y gubernamentales en el sector ambiental. Por este motivo, realizar una aportación para clarificar el uso de la teoría de la gobernanza en este sector puede beneficiar a las futuras investigaciones en la materia.

\section{Bibliografía}

Aguilar-Villanueva, Luis F. (2006), Gobernanza y gestión pública, Fondo de Cultura Económica, México.

Aguilar-Villanueva, Luis F. (2010), Gobernanza: el nuevo proceso de gobernar, Fundación Friedrich Naumann para la Libertad, México.

Antinori, Camile y Gordon Rauser (2007), "Collective choice and community forestry management in Mexico: an empirical analysis", The Journal of Development Studies, 43 (3), Taylor and Francis Group, London, pp. 512-536. 
Barsimantov, James A. (2009), "Vicious and virtuous cycles and the role of external non-government actors in community forestry in Oaxaca and Michoacán, Mexico", Human Ecology, 38 (1), Springer, New York, pp. 49-63.

Bassols, Mario (2011), “Gobernanza: una mirada desde el poder”, en Mario Bassols y Cristóbal Mendoza (coords.), Gobernanza; teoría y prácticas colectivas, Anthropos Editorial-uAm unidad Iztapalapa, Barcelona, pp. 7-35.

Basurto, X. (2005), "How locally designed access and use controls can prevent the tragedy of the commons in a mexican small-scale fishing community", Society and Natural Resources, 18 (7), Taylor \& Francis Group, London, pp. 643-659.

Basurto, X. (2008), "Biological and ecological mechanisms supporting marine self-governance: the seri callo de hacha fishery in Mexico", Ecology and Society, 13 (2), Acadia University, Wolfville, pp. 2-20.

Bobadilla, Mariana, Ileana Espejel, Francisco Lara-Valencia, Saúl ÁlvarezBorrego, Sophie Foucat-Ávila y José Luis Fermán-Almada (2013), "Esquemas de evaluación para instrumentos de política ambiental", Política y Cultura, 40, uam unidad Xochimilco, México, pp. 99-122.

Brenner, Ludger (2010), "Gobernanza ambiental, actores sociales y conflictos en las áreas naturales protegidas mexicanas", Revista Mexicana de Sociología, 72 (2), Universidad Nacional Autónoma de México, México, pp. 283-310.

Brenner, Ludger (2011), "Retos para la gobernanza ambiental en México: el caso de la Reserva de la Biosfera Mariposa Monarca", en Mario Bassols y Cristóbal Mendoza (coords.), Gobernanza. Teoría y prácticas colectivas, Anthropos Editorial-UAm unidad Iztapalapa, Barcelona, pp. 141-175.

Brenner, Ludger, y Anne Cristina de la Vega-Leinert (2014), "La gobernanza participativa de áreas naturales protegidas. El caso de la Reserva de la Biosfera El Vizcaíno", Región y Sociedad, 26 (59), El Colegio de Sonora, Hermosillo, pp. 183-213. 
Coleman, Mathew (2007), "Immigration geopolitics beyond the Mexicous border", Antipode, 39 (1), Editorial Board of Antipode, Manchester, pp. 54-76.

Concepción-Montiel, Luis Enrique (2010), "Gobernanza y democracia en América Latina en un contexto de globalización", en Luis Concepción-Montiel y Patricia Moctezuma-Hernández (coords.), Gobernanza global y democracia, Universidad Autónoma de Baja California, Mexicali, pp. 301-321.

Domínguez-Serrano, Judith (2010), "El acceso al agua y saneamiento: un problema de capacidad institucional local", Gestión y Politica Pública, 19 (2), Centro de Investigación y Docencia Económicas, A. C., México, pp. 311-350.

Foladori, Guillermo (2002), "Avances y limites de la sustentabilidad social”, Economia, Sociedad y Territorio, III (12), El Colegio Mexiquense, A. c., Zinacantepec, pp. 621-637.

Foladori, Guillermo (2009), "La gobernanza de las nanotecnologías", Sociológica, 24 (71), UAm unidad Azcapotzalco, México, pp.125-153.

Filibi-López, Igor (2010), "Democracia postmoderna: gobernanza o el retorno de lo político", en Luis Concepción-Montiel y Patricia Moctezuma-Hernández (coords.), Gobernanza global y democracia, Universidad Autónoma de Baja California, Mexicali, pp. 107-128.

Fox, Jonathan (1995), "Governance and rural development in Mexico: state intervention and public accountability", The Journal of Development Studies, 32 (1), Taylor \& Francis Group, London, pp. 1-30.

Funtowicz, Silvio y Jerome Ravetz (1993), "Science for the postnormal age", Futures, 25, Elsevier, Maryland Heights, pp. 735-755.

Guarneros-Meza, Valeria (2011), "Localismo, neoliberalismo y poder: élites urbanas y prácticas culturales en Polonia y México", en Mario Bassols y Cristóbal Mendoza (coords.), Gobernanza. Teoría y prácticas colectivas, Anthropos Editorial-UAm unidad Iztapalapa, Barcelona, pp. 177-207. 
Guzmán-Chávez, Mauricio Genet (2006), "Biodiversidad y conocimiento local: del discurso a la práctica basada en el territorio", Espiral, Estudios sobre Estado y Sociedad, 12 (37), Universidad de Guadalajara, Guadalajara, pp. 145-176.

Hyun, Karen (2005), "Transboundary solutions to environmental problems in the gulf of California large marine ecosystem", Coastal Management, 33 (4), Taylor \& Francis Group, London, pp. 435-445.

Klooster, Dan (2006), "Environmental certification of forests in Mexico: the political ecology of a nongovernmental market intervention", Annals of the Association of American Geographers, 96 (3), Taylor \& Francis Group, London, pp. 541-565.

Kooiman, Jan (1993), Modern governance. New government-society interactions, Sage publications, London.

Kooiman, Jan (2003), Governing as governance, Sage publications, London.

Leff, Enrique (2000), "Pensar la complejidad ambiental", en Enrique Leff (coord.), La complejidad ambiental, Siglo XXI, México, pp.7-53.

Lezama, José Luis (2010), "Sociedad, medio ambiente y política ambiental”, en José Luis Lezama y Boris Graizbord (coords.), Los grandes problemas de México, El Colegio de México, México, pp. 23-59.

Ludwig, Donald, Marc Mangel y Brent Haddad (2001), "Ecology, conservation, and public policy", Annual Review of Ecology and Systematics, 32, Annual Reviews, Palo Alto, pp. 481-517.

Martínez, Nain y Refugio Chávez-Ramírez (2014), "Nuevos agentes en la gestión ambiental: el caso de las organizaciones de la sociedad civil en Ensenada”, Estudios Fronterizos, 15 (29), Universidad Autónoma de Baja California, Mexicali, pp. 85-123.

Monsiváis C., Alejandro (2006), "Rendición de cuentas democrática y justificaciones públicas: una concepción deliberativa de la calidad de la democracia”, Sociológica, 21 (62), uAm unidad Aztcapotzalco, México, pp. 13-41.

Moreno-Mena, José Ascención (2010), "Procesos de gestión pública innovadora: el caso de los módulos de atención a migrantes en Baja 
California”, Estudios Fronterizos, 11 (21), Universidad Autónoma de Baja California, Mexicali, pp. 143-170.

Morse, Wayde Cameron, Max Nielsen-Pincus, Jo Ellen Force y J. D. Wulfhorst (2007), "Bridges and barriers to developing and conducting interdisciplinary graduate-student team research", Ecology and Society, 12 (2), pp. 1-14, <http://www.ecologyandsociety.org/vol12/iss2/art8/>, 15 de diciembre de 2011.

Palerm-Viqueira, Jacinta (2009), "Governance and management of irrigation systems”, Water Policy, 11 (3), IWA Publishing, London, pp. 330-347.

Pardo, María del Carmen (2004), De la administración pública a la gobernanza, El Colegio de México, México.

Paré, Luisa y Tajín Fuentes (2010), Gobernanza ambiental y politicas públicas en áreas naturales protegidas. Lecciones desde los Tuxtlas, Universidad Nacional Autónoma de México, México.

Paz-Salinas, María Fernanda (2007), "De áreas naturales protegidas y participación: convergencias y divergencias en la construcción del interés público", Nueva Antropología, XXI (68), Universidad Nacional Autónoma de México, México, pp. 51-74.

Paz-Salinas, María Fernanda (2008), “Tensiones de la gobernanza en el México rural", Política y Cultura, 30, uAm Unidad Xochimilco, México, pp. 193-208.

Peters, Guy B. (2004), "Cambios en la naturaleza de la administración pública. De las preguntas sencillas a las respuestas dificíles", en María del Carmen Pardo (coord.), De la administración pública a la gobernanza, El Colegio de México, México, pp. 69-95.

Peters, Guy B. y Jean Pierre (2005), “¿Por qué ahora el interés por la gobernanza?”, en Agustí Cerrillo i Martínez (coord.), La gobernanza hoy: 10 textos de referencia, Instituto Nacional de Administración Pública, Madrid, pp. 37-56.

Porras, Francisco (2011), “¿Sistema, continuum, modo o marco general? La anglo-gobernanza en México”, en Mario Bassols y Cristóbal 
Mendoza (coords.), Gobernanza. Teoría y prácticas colectivas, Anthropos Editorial-uam unidad Iztapalapa, Barcelona, pp. 67-69.

Prats, Joan (2005), "Modos de gobernación de las sociedades globales", en Agustí Cerrillo i Martínez (coord.), La gobernanza hoy: 10 textos de referencia, Instituto Nacional de Administración Pública, Madrid, pp.145-201.

Racelis, Alexis E. y James A. Barsimantov (2008), “The management of small diameter, lesser-known hardwood species as polewood in forest communities of central Quintana Roo, Mexico", Journal of Sustainable Forestry, 27 (1), Taylor \& Francis Group, London, pp. 122-144.

Reyes-Orta, Marisa, Myriam Cardozo-Brum, Concepción ArredondoGarcía, Hugo Méndez-Fierros e Ileana Espejel (2013), "Análisis del sistema de evaluación de un programa ambiental de la política mexicana: el PRODERS su transformación al PROCODES”, Investigación ambiental Ciencia y política pública, 5 (2), SemarnatINECC, México, pp. 44-61.

Rhodes, R.A.W. (1996), "The new governance: governing without government”, Political Studies, XLIV, Blackwell Publishers, Oxford, pp. 652-667.

Rivera-Arriaga, Evelia e Isaac A. Azuz-Adeath (2010), "La gobernanza de las costas y océanos de México en un clima cambiante", en Evelia Rivera-Arriaga, Isaac Azuz-Adeath, Leticia Alpuche Gual y Guillermo J. Villalobos-Zapata (eds.), Cambio climático en México: un enfoque costero y marino. Elementos ambientales para tomadores de decisiones, Universidad Autónoma de CampecheCETyS-Universidad-Gobierno del Estado de Campeche, México, pp. 739-772.

Rodríguez-González, Román y Argimiro Rojo-Salgado (coords.) (2009), Ordenación y gobernanza en las áreas urbanas gallegas, Netbiblo, Oleiros.

Rodríguez-Garavito, César A. (2008), "Gobernanza global y derechos laborales: códigos de conducta y luchas antimaquila en las fábricas globales del vestido en México y Guatemala", Revista de Derecho Privado, 40, Universidad de los Andes, Bogotá, pp. 1-33. 
Rojo-Salgado, Argimiro (2010), "Globalización y crisis de la política: la necesidad de instaurar el espacio público”, en Luis ConcepciónMontiel y Patricia Moctezuma-Hernández (coords.), Gobernanza global y democracia, Universidad Autónoma de Baja California, Mexicali, pp. 129-146.

Salcido-Ruiz, Silvia, Peter-R. W. Gerritsen y Luis Manuel Martínez (2010), "Gobernanza del agua a nivel local: estudio de caso en el municipio de Zapotitlán de Vadillo, Jalisco", El Cotidiano, 162, uAM unidad Azcapotzalco, México, pp. 83-89.

Tortajada, Cecilia (2010), "Water governance: a research agenda", Water Resources Development, 26 (2), Taylor \& Francis Group, London, pp. 309-316.

Villada-Canela (2013), "El rol de la información la participación pública en la planeación ambiental", Investigación ambiental Ciencia y política pública, 5 (2), Semarnat-INECC, México, pp. 17-26.

Wilder, Margaret (2010), "Water governance in Mexico: political and economic aperatures and a shifting state-citizen relationship", Ecology and Society, 15 (2), artículo 22, pp. 1-15, <http://www. ecologyandsociety.org/vol15/iss2/art22/>, 25 de noviembre de 2011.

Wilshusen, Peter R. (2010), "The receiving end of reform: everyday responses to neoliberalisation in southeastern Mexico", Antipode, 42 (3), Editorial Board of Antipode, Manchester, pp 767-799.

Recibido: 26 de julio de 2012.

Reenviado: 3 de julio de 2013. Aceptado: 7 de febrero de 2014 .

Nain Martínez. Mexicano. Es maestro en ciencias en manejo de ecosistemas de zonas áridas por la Universidad Autónoma de Baja California. Actualmente es director de Tierra Colectiva: Ciudadanía, Género y Medio Ambiente, A. c. Sus líneas de investigación son el manejo integrado de ecosistemas, la participación ciudadana en el sector ambiental y la política de conservación. Su primera publicación, en coautoría, "Nuevos agentes en la gestión ambiental: el caso de las organizaciones de la sociedad civil en Ensenada”, Estudios Fronterizos, 15 (29), Universidad Autónoma de Baja California, Mexicali, pp. 85-123 (2014). 
Ileana Espejel. Mexicana. Es doctora en ecología botánica por la Universidad de Uppsala, Suecia. Actualmente es profesora-investigadora de la Facultad de Ciencias de la Universidad Autónoma de Baja California. Es miembro del Sistema Nacional de Investigadores, nivel II. Sus líneas de investigación actuales son el manejo integrado de ecosistemas, en especial los áridos y los costeros, y los estudios interdisciplinarios. Entre sus últimas publicaciones, siempre en coautoría con sus alumnos y colegas, se encuentran: "Scenarios of vulnerability in coastal municipalities of tropical Mexico: An analysis of wetland land use", Ocean \& Coastal Management, (89), Elsevier, Maryland Heights, pp. 11-19 (2014); "Using traditional ecological knowledge to improve holistic fisheries management: transdisciplinary modelling of a lagoon ecosystem of southern Mexico", Ecology and Society, 18 (2), artículo 6, Acadia University, Wolfville, (2013); "Environmental quality and equity in the Human Development Index: an integrated index for the Local Agenda 21 case study", Journal of Economics and Sustainable Development, 3 (12), International Knowledge Sharing Platform, New York, pp. 39-51 (2012). 